\title{
OXIDATION MANAGEMENT OF WHITE WINES USING CYCLIC VOLTAMMETRY AND MULTIVARIATE PROCESS MONITORING
}

\author{
R. Martins ${ }^{1}$, F. Bento ${ }^{2}$, D. Geraldo ${ }^{2}$, V.V. Lopes ${ }^{3}$, T. I. M. S. Lopes ${ }^{4}$, C. Oliveira ${ }^{4}$ and A. C. \\ SILVA FERREIRA ${ }^{4 *}$
}

1 BioInformatics Research Group, Molecular and Environmental Biology, Campus de Gualtar, Braga

${ }^{2}$ Departamento de Química, Universidade do Minho, Campus de Gualtar, Braga

3 Laseeb Research Group, Institute for Systems and Robotics, IST, Lisboa

${ }^{4}$ Escola Superior de Biotecnologia, Universidade Católica Portuguesa, Rua Dr. António

Bernardino de Almeida, 4200-072 Porto

E-mail : acferreira@esb.ucp.pt

Keywords: Wine Oxidation, process analytical technology, Resistance to Oxidation

Several simple mechanisms both enzymatic and non-enzymatic are responsible for differences in sensory perception throughout the winemaking process and, due to interaction and overlapping, they account for the compositional complexity perceived as changes in color, taste and flavor ultimately influencing final product quality. Among these mechanisms the redox phenomenon stands out with particular relevance due to the large impact on the sensory quality of white wines, and consequently on the product shelf-life (1).

From an industrial point of view, it should be noted that there is no systematic way to predict the shelf life of bottled white wines. Hence, the aim of this work was to quantify the white wine fraction with higher antioxidant power, and in what extent this value could be related with white wine oxidative spoilage. For this purpose, three parameters were measured: (i) the index of degradation (ID) evaluated by sensorial analysis (1), (ii) the levels of substances responsible for "off flavors" in white wines (2) (iii) the "resistance to oxidation" measured by cyclic voltammetric (3).

Voltammetric studies were carried out in wines in different stages of degradation normal and submitted to a forced aged protocol, in order to check the potential of the voltammetric techniques for the evaluation the degree of wine degradation. Current intensities and charges were measured along time at the potential range where antioxidants are electroactive. It was observed a high correlation between electrochemical measurements with both chemical and sensorial data (3).

Finally multivariate process monitoring or process analytical technology (PAT) was employed to research the causes of the oxidative processes by an empirical diagnostic tool, the Multivariate Control Chart (MCC) (4).

MCC was constructed based on the singular value decomposition of the oxidative voltammogram of non oxidative spoiled young wines. Thereafter, aged and abused wines were plotted into de MCC, those inside the control limits can be considered similar to the ones used to develop the control chart (5). An effective way to determine the influence of variables on the projected scores is by estimating the Contribution Plot (CP). This feature is 
useful to identify the oxidation potentials responsible for out the confidence intervals sample prediction.

These promising results points to the possibility of online applications of electrochemical sensors for managing the oxidation of wines in stock establish the shelf-life and decide the logistic pathways for each specific production.

(1) Oliveira C, Silva Ferreira A C, Guedes de Pinho P, Hogg T (2002). Development of a potentiometric method in order to measure the "Resistance to Oxidation" of white wines and the "Antioxidant Power" of their constituents. J. of Agric. Food Chem. 50, (7) 2121-2124.

(2) Silva Ferreira A C, Guedes de Pinho P, Rodrigues P, Hogg T. (2002). Kinetics of oxidative degradation of white wines and how they are effected by selected technological parameters. $J$. Agric. Food Chem. 50, (21) 5919-5924.

(3) Rodrigues A, Silva Ferreira A C, Guedes de Pinho P, Bento F, Geraldo D, (2007) Resistance to Oxidation of White Wines Assessed by Voltammetric Means J. Agric and Food Chemistry (in press)

(4) Qin, SJ. (2003). Statistical process monitoring: basics and beyond Journal of Chemometrics, $17,480-502$.

(5) Miller P, Swanson RE, Heckler CF. (1993). Contribution plots: the missing link in multivariate quality control. International Journal of Applied Computational Science 9, 775-792.

Indicate your preference:

oral communication

poster presentation 